\title{
A Hybrid Planning Method for Transmission Networks in a Deregulated Environment
}

\author{
Zhao Xu, Student Member, IEEE, Zhao Yang Dong, Member, IEEE, and Kit Po Wong, Fellow, IEEE
}

\begin{abstract}
The reconstruction of power industries has brought fundamental changes to both power system operation and planning. This paper presents a new planning method using a multi-objective optimization (MOOP) technique, as well as human knowledge, to expand the transmission network in open-access schemes. The method starts with a candidate pool of feasible expansion plans. Consequent selection of the best candidates is carried out through a MOOP approach, of which multiple objectives are tackled simultaneously, aiming at integrating the market operation and planning as one unified process in context of a deregulated system. Human knowledge has been applied in both stages to ensure the selection with practical engineering and management concerns. The expansion plan from MOOP is assessed by reliability criteria before it is finalized. The proposed method has been tested with the IEEE 14-bus system, and relevant analyses and discussions have been presented.
\end{abstract}

Index Terms-Electricity market, goal programming, multi-objective optimization, transmission planning.

\section{NOMENCLATURE}

$F_{i} \quad$ Individual objective in transmission planning.

$C \quad$ Construction cost vector of new transmission lines.

$\eta_{i j} \quad$ Integer, the actual of new circuit addition in transmission route $i-j$ (bus $i-j$ ).

$\eta_{i j}^{M a x} \quad$ Maximum addition in transmission route $\mathrm{i}-\mathrm{j}$.

$P_{G i}, \quad$ Real and reactive power outputs of generator $i$.

$Q_{G i}$

$P_{D i}$,

$Q_{D i}$

$f_{i j}$

$d_{i j}$

$r_{i j}$

$S_{i j}^{f}$

$S$

$S_{i j}^{M a x}$

$\delta_{i}$

$\delta_{i}$

$\boldsymbol{Y}$

$y_{i j}^{0}$

$y_{i 0}$

$\tau_{i j}$

Forecasted real and reactive demands at bus $i$ in planning horizon.

Real power flow in transmission route $i-j$.

Length of transmission line $i-j$.

Pre-determined transmission tariff per unit capacity of the line in $\$ / \mathrm{MW}$-mile.

Apparent power flow of transmission line $i-j$

Feasible searching region of optimal solutions.

Capacity of transmission line $i-j$.

Voltage angle at bus $i$.

Bus admittance matrix of the system.

Initial admittance of branch $i-j$.

Shunt admittance at bus $i$.

new circuit admittance of branch $i-j$;

Manuscript received January 27, 2005; revised September 16, 2005. Paper no. TPWRS-00022-2005.

Z. Xu and Z. Y. Dong are with the School of Information Technology and Electrical Engineering, the University of Queensland, St. Lucia, QLD 4072, Australia (e-mail: xuzhao@itee.uq.edu.au; zdong@itee.uq.edu.au).

K. P. Wong is with the Department of Electrical Engineering, the Hong Kong Polytechnic University, Hung Hom, Kowloon, Hong Kong (e-mail: eekpwong@polyu.edu.hk).

Digital Object Identifier 10.1109/TPWRS.2006.873134 $\theta_{\text {in }} \quad$ Angle of element $Y_{\text {in }}$ in $\boldsymbol{Y}$.

$a_{i}, b_{i}, \quad$ Constants in generator $i$ 's cost function.

and $c_{i}$

$h \quad$ Planning year.

$\mu \quad$ Discount rate.

$N_{G} \quad$ Set of generators.

$N \quad$ Set of system bus.

$N_{D} \quad$ Set of loading scenarios considered in planning.

$\Omega \quad$ Set of candidate transmission lines for expansion selected at initial stage of planning.

$\zeta_{\mathrm{i}}, \tau_{i} \quad$ Positive and negative deviations of $i$ th objective in goal programming.

$\alpha_{\mathrm{i}}, \beta_{\mathrm{i}} \quad$ Weights for deviations in goal programming.

$t_{i}$

$T_{k}$

OVL

Predefined target in goal programming.

Duration of loading scenario $k$ in planning.

Overload in transmission lines.

$O c_{l} \quad$ Overload relieve per unit line construction cost.

$\alpha$

s

$f \quad$ Vector of active-power flows through the lines.

$g \quad$ Vector of generated active power.

$L \quad$ Vector of load curtailments caused by lack of transmission capacity.

$D \quad$ Vector of predicted loads.

$U \quad$ Array of ones.

$x_{i j} \quad$ Total new circuit susceptance added to branch $i-j$.

$x_{i j}^{0} \quad$ Initial susceptance in branch $i-j$.

$f_{i j}^{M a x} \quad$ Real power flow limit of branch $i-j$.

$g^{M a x}$

$r l_{i j}$

Vector of maximum generation capacity.

Circuit susceptance.

\section{INTRODUCTION}

$\checkmark \mathrm{TE}$ ongoing deregulation of power systems from vertically integrated monopoly into free, competitive markets aims at maximizing the overall social welfare [1], [2]. Not only has the deregulation developed in the generation sector, with emergence of independent generation companies and competitive electricity markets, but also in the retail supply sector of both transmission and distribution.

Since the transmission network is of vital importance in connecting individual generators with the system and enabling necessary competitions in electricity supply, various deregulation approaches have been adopted in different countries. However, the reform in transmission network involves a number of complexities, and many new issues have surfaced, such as transmission costing and pricing, payment allocation, power wheeling and congestion management, available power transfer capabilities, the natural monopoly status of transmission networks and 
transmission rights, etc. [1]. Among them, the transmission network expansion planning (TNEP) poses particularly a difficult question in the newly deregulated environment, which is the main focus of this paper.

Traditionally, the system operator is solely responsible for system operation and planning. By setting their objectives separately, the operators used to run the systems to minimize costs or losses and plan the expansion to minimize investments in a centralized manner while fulfilling the obligation of energy supply with reliability requirements [3], [4]. The transmission planning problem, though dynamic in nature, is often simplified by the planning engineer to a static optimization model, minimizing the total investment of network expansion for a single future situation, subject to operational constraints [3]. The typical model appeared in most technical literatures, for such static transmission network expansion planning (STNEP) is formulated as follows [5]-[10]:

$$
\begin{array}{cl}
\text { Minimize } & \\
& J=\boldsymbol{C}^{T} \boldsymbol{\eta}+\alpha \boldsymbol{U}^{T} \boldsymbol{L} \\
\text { subject to } & \boldsymbol{s}^{T} \boldsymbol{f}+\boldsymbol{g}+\boldsymbol{L}=\boldsymbol{D} \\
& f_{i j}-\left(x_{i j}^{0}+x_{i j}\right)\left(\delta_{i}-\delta_{j}\right)=0 \\
& \left|f_{i j}\right|-x_{i j} \xi_{i j} \leqslant x_{i j}^{0} \xi_{i j} ; \xi_{i j}=\frac{f_{i j}^{M a x}}{x_{i j}^{0}} \\
& 0 \leqslant \boldsymbol{g} \leqslant \boldsymbol{g}^{M a x} ; 0 \leqslant \boldsymbol{L} \leqslant \boldsymbol{D} \\
& x_{i j}=\eta_{i j} r l_{i j} ; 0 \leqslant \eta_{i j} \leqslant \eta_{i j}^{M a x} \forall(i, j) \in \Omega .
\end{array}
$$

The planning objective (1) considers both the investments of new transmission lines and the cost of the loss of load, i.e., load curtailments due to inadequate transmission capacity. The solution of the objective is an expansion plan of minimum investment cost while ensuring the loss of load equals to zero. Constraints (2) and (3) are the linearized dc-flow model for the future system, which can be replaced by the ac-flow model for better accuracy. Constraints (4) and (5) give the operational limits, while (6) specifies the range of the decision variables.

The least-cost transmission planning model presented above is based on the fact that the power system is a vertically integrated system owned, operated, and planned by a central operator. However, this is no longer the case in the deregulated environment with many new key stakeholders of power utilities appearing, such as transmission companies (Transco), power plant owner, power marketers and load-serving entities, shareholders of companies that build new facilities, regulator and investor from financial markets, etc. [11]-[13]. These new entities are certainly getting involved in the transmission planning, and their objectives would be rather market-oriented, in which investment and profit prospects would be equally concerned. Since the physical topology of transmission network, and hence the relevant transmission tariffs, has a natural influence over the operation of electricity markets, therefore, the selection of the expansion plan can affect significantly the future transmission profit, as transmission and various ancillary services become commodities. Obviously, the traditional least-cost planning method does not reflect this economic signal and is no longer appropriate in this context.

Recognizing the inherent limitations of the traditional planning approach in light of changing electric power industries, we propose a new planning method for TNEP to meet the challenges introduced by the deregulation. Based on the traditional method, the proposed method integrates the operation and planning as a unified process and seeks to not only minimize the total investment but also to maximize the overall social benefit from the expansion to be consistent with the ultimate goal of the deregulation. The new method starts with an initial candidate pool of feasible expansion plans. Subsequently, the selection of the best candidate subset is carried out through a MOOP approach using the goal programming method [14], though human knowledge is needed in certain stages to ensure the selection with practical engineering and management concerns. To enhance the system reliability, the resultant expansion plan from MOOP has been further assessed by the " $N-1$ " criteria. The proposed method has been tested with the IEEE 14-bus system, and results are presented with relevant analyses and discussions.

\section{OVERVIEW OF THE PlanNING Method}

Power system planning is a complex process that requires a significant amount of work, involving major stages such as system reliability assessment, forecasting of demand and fuel prices, and security assessment. The main focus of this paper is on a new transmission planning approach with multi-objective optimization techniques, subject to security assessment. Other works, such as forecasting, reliability assessment, and generation expansion, are assumed to have been dealt with properly. Such assumptions will not lose the generosity of this new method. The authors have developed new advanced techniques for power system load and price forecasting that are readily applicable to the planning task [15], [16].

Fig. 1 presents the structure of the proposed method, in which the early stages of planning, such as demand forecasting and reliability assessment, are not included. The method has three stages, including the initial formulation of the candidate pool for expansion, followed by a multi-objective optimization approach, subject to constraints, and the final security assessment of the expansion plan from MOOP using the " $N-1$ " criteria.

\section{A. Initialization}

The initial candidate pool of transmission lines is formulated at this stage based on the given system information. Human knowledge is used in this stage to ensure rationality of the candidate line selection with practical engineering and management concerns.

\section{B. Expansion Plan Selection by MOOP}

The selection from the candidate pool is carried out through a multi-optimization approach using the goal programming method, subject to a list of constraints. Again, the planner's preference, as the human knowledge, is needed at this stage. 


\section{Security Assessment}

The resultant optimal expansion plan from the last stage is, however, without system security constraints. Therefore, the final step assesses the security of the plan by " $N-1$ " criteria. A list of contingencies generated by contingency screening is simulated sequentially to assess the expanded system. Should overload happen in any transmission line, a best candidate is selected from the rest of the pool to enhance the reliability. The assessment is carried out recursively until no overload happens and the optimal expansion plan with security concern is obtained. Detailed description of each stage and the techniques associated will be given in the following sections.

\section{PRoblem Formulation AND Mathematical Modeling}

\section{A. Formulation of Candidate Pool}

In the proposed planning method, the initial candidate pool for expansion is formulated based on the information of the given system, such as the generation and transmission capacity, the estimated transmission tariffs, the planning scope and corresponding forecasted system demand, etc. To ensure the initialization with practical engineering and management concerns, human knowledge is applied with several rules employed. These rules may include but not limited to the following:

- network redundancy;

- environmental factors;

- financial limits;

- estimated construction periods of transmission lines and the time horizon of planning.

The formulation of the candidate pool can start from deciding the planning horizon and corresponding market forecasts. Subsequently, market simulation can be carried to identify insufficient transmission capacity. The pool is then formulated to enhance transmission with redundancy for system reliability. Next, by examining investments and construction periods of individual lines, a number of candidates can be dropped at this stage to avoid excessive workload in the following steps. By taking into account the environmental factors, further reduction of the pool can be made, for example, to avoid a line construction through an important natural reserve.

\section{B. Modeling of Network Expansion}

To investigate the transmission planning problem, a mathematical model representing the decision-making process should be carefully built. First of all, the candidate transmission lines need to be modeled. In this paper, an array is used to keep track of the candidate lines in each transmission corridor [5], [9]. Inside the array, an element represents the number of lines to expand in corresponding transmission route. Fig. 2 illustrates the array representation of network expansion, where the transmission network is partially presented, from branch 15 to 18 . In Fig. 2, the array indicates that there is one line each to expand on routes 15 and 17; also, there are two lines to expand on route 16. Obviously, an array with all zero elements, e.g., route 18, represents the original network before expansion.

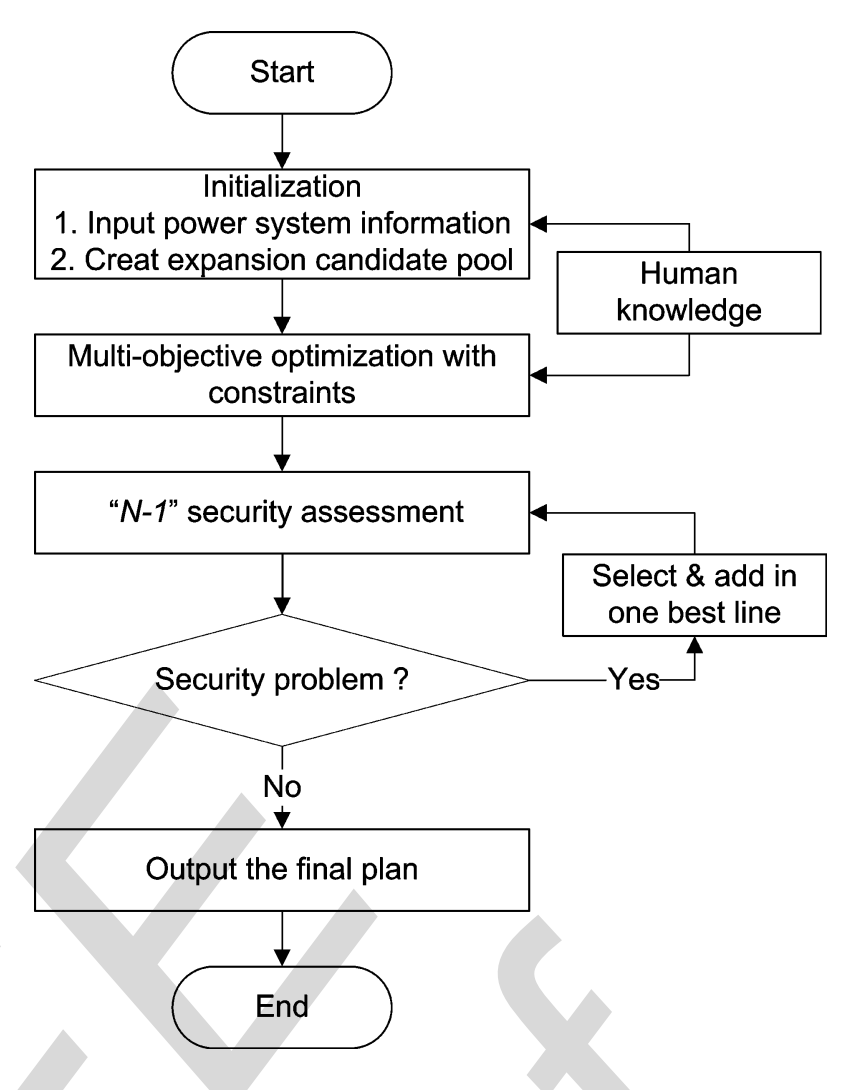

Fig. 1. Flowchart of the hybrid planning scheme for TNEP.

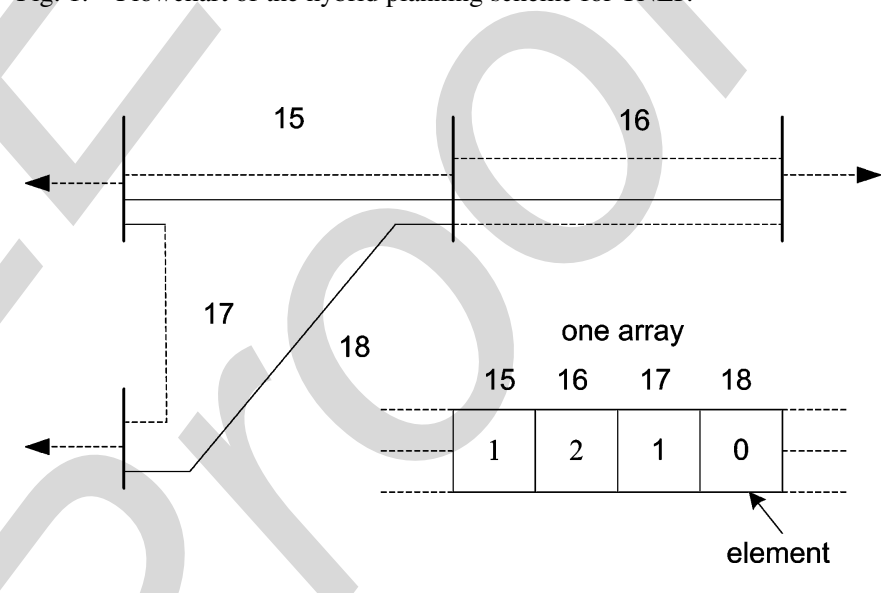

Fig. 2. Modeling of network expansion using arrays.

\section{Planning Objectives}

All relevant factors should be included into the planning model, such as the investment and expected demand. To comprehensively meet the challenge of market economy, a multi-objective TNEP model is proposed. It basically maximizes the overall benefit of expansion (BOE) while minimizing the overall investment, subject to system power flow, security constraints, etc. In addition, minimization of the generation cost is included since the deregulated electricity market should be dispatched at the least generation cost, if the system marginal price (SMP) auction is employed in market clearing [17]. To account for the time value of money, individual objective is converted to its net present value (NPV), assuming the investment happens at the beginning of the planning year $(h)$ and 
new circuits are immediately ready to serve. It also assumes the transmission and generation incomes happen at the middle of the planning year $(h+0.5)$. The objectives of planning can be written as

$$
\begin{aligned}
& \text { Minimize } \\
& \begin{aligned}
F_{1} & =\frac{\boldsymbol{C}^{\boldsymbol{T}} \boldsymbol{\eta}}{(1+u)^{h}} \\
F_{2} & =\frac{f_{2}\left(\boldsymbol{P}_{G}\right)}{(1+\mu)^{h+0.5}} \\
& =\frac{\left[\sum_{i \in N_{G}}\left(a_{i}+b_{i} P_{G i}+c_{i} P_{G i}^{2}\right)\right]}{(1+\mu)^{h+0.5}}
\end{aligned}
\end{aligned}
$$

Maximize

$$
\begin{aligned}
F_{3} & =\frac{f_{3}(\boldsymbol{f}, \boldsymbol{\eta}, \boldsymbol{r}, \boldsymbol{d})}{(1+\mu)^{h+0.5}} \\
& =\frac{\left[\sum_{(i, j) \in \Omega} f_{i j} r_{i j} d_{i j} \frac{\eta_{i j}}{\eta_{i j}+1}\right]}{(1+\mu)^{h+0.5}}
\end{aligned}
$$

Subject to

$$
\begin{aligned}
& P_{G i}-P_{D i}=\sum_{i=1}^{N}\left|Y_{i n} V_{i} V_{n}\right| \cos \left(\theta_{i n}+\delta_{n}-\delta_{i}\right) \\
& Q_{G i}-Q_{D i}=-\sum_{i=1}^{N}\left|Y_{i n} V_{i} V_{n}\right| \sin \left(\theta_{i n}+\delta_{n}-\delta_{i}\right) \\
& S_{i j}^{f} \leqslant S_{i j}^{M a x} \\
& V_{i}^{M i n} \leqslant V_{i} \leqslant V_{i}^{M a x} \\
& Q_{G i}^{M i n} \leqslant Q_{G i} \leqslant Q_{G i}^{M a x} \\
& P_{G i}^{M i n} \leqslant P_{G i} \leqslant P_{G i}^{M a x} \\
& Y_{i j}=-\left(y_{i j}^{0}+\eta_{i j} \tau_{i j}\right) \quad(i \neq j) \\
& Y_{i i}=y_{i 0}+\sum_{j \in i}\left(y_{i j}^{0}+\eta_{i j} \tau_{i j}\right) \quad(i \neq j) \\
& 0 \leqslant \eta_{i j} \leqslant \eta_{i j}^{M a x} \forall(i, j) \in \Omega
\end{aligned}
$$

where $F_{1}$ and $F_{2}$ are the total investment of expansion and the generation cost of the system, respectively, and $F_{3}$ is the profit prospect of the expanded transmission lines, based on the MW-mile method, in which the transmission cost is recovered by multiplying the actual power flow, the distance, and the pre-determined unit cost of each transmission line [18], [19]. Equation (9) basically calculates the profits of the expanded lines in all transmission corridors, assuming lines in one corridor have the same distance and profit rate. The advantage of the MW-mile pricing method used in (9) is to allocate the costs based on actual system usage as closely as possible, which is superior to other methods, such as post-stamp and contract path methods [19]. Constraints (10)-(18) are similar to those in ac optimal power flow, while (16) and (17) simply update the network admittance matrix with expansion. Equation (18) requires transmission line expansion within the bounds of maximum line addition. It should be noted that the transmission service considered is restricted to real power transmission only. Other ser- vices, i.e., various ancillary services, are not taken into account for simplicity. Such services can be considered in planning if in need; however, since real power is of primary concern in expansion planning and the security assessment is included as well, our approach will not lose the generosity as a framework of TNEP.

In (9), the estimated transmission profit can be considered as the difference between transmission customer's payment and service provider's cost in future transactions, which is actually the social benefit from the transmission deals. Maximizing the social benefits is undoubtedly the first priority in the marketplace, which certainly should be reflected into the planning stage, if considering the market planning and operation as one integrated process. Similarly, the investment of expansion can also be considered as the "costs" of the provider, assuming they may not be fully recovered by the profit. Thus, the planning objective is formulated as maximizing the benefit of expansion, namely, the profit, while minimizing the planning costs. Moreover, it would be realistic to consider transmission transactions in context of overall system dispatching. Therefore, a least-cost dispatching objective is included in (8), which should produce an equivalent dispatching as a typical SMP-based market does, assuming all market participants are rational [17]. The proposed planning method actually seeks to maximize the overall social benefit from expansion by incorporating various factors of interests in horizon into one optimization approach and tackling multiple objectives simultaneously.

\section{Multi-Objective Optimization}

The planning model actually formulates a multi-objective optimization problem with incompatible objectives, subject to a list of constraints. One possible solution is to directly combine all objectives into one by using the weighted sum method [14]. However, this method suffers from many disadvantages, such as the difficulty in finding the Pareto-optimal solution, i.e., the genuine optimal solution for all objectives, though with great simplicity. The "ideal point" or weighted sum metric method is another option, which minimizes the distance between the practical solution and the given ideal solution. Though this method can guarantee finding the Pareto-optimal solution if using highorder distance function, the computational cost will be quite high simultaneously [14].

The goal programming method is an efficient MOOP technique and is chosen in our approach. It features lesser computational complexity and greater flexibility in dealing with various types of optimization problems, such as the "equal to" and "greater and equal to" problems. It attempts to find solutions that attain the predefined targets or minimize the deviation from targets if such solutions are not achievable. The expansion planning model of (7)-(9) can be defined as an "equal to" type problem, which is formulated as

$$
\begin{array}{ll}
\text { goal } & f_{i}(x)=t_{i} \\
& x \in S, \quad i=1, \ldots, M
\end{array}
$$

where $S$ is the feasible search region, and $t_{i}$ is the predefined target for the individual planning objective. The ideal targets can 
be derived by estimation or by optimizing the objectives independently prior to MOOP, which is applied in our case study [14]. In addition, since the objectives can be of different orders of magnitude, normalization of objectives is needed [14]. There are two ways for normalization. The first method requires solving objectives to find their minima and maxima. The second method multiplies objectives with proper constant values and normalizes them approximately to the same order of 10 . The second method is adopted in our case study [14]. Subsequently, a composite objective with deviations from each of the $M$ objectives is formulated by using the weighted goal programming approach [14]

$$
\begin{array}{cl}
\text { Minimize } & \sum_{i=1}^{M} \alpha_{i} \zeta_{i}+\beta_{i} \tau_{i} \\
\text { subject to } & f_{i}(x)-\zeta_{i}+\tau_{i}=t_{i} \\
& x \in S \\
& \zeta_{i}, \tau_{i} \geqslant 0, i=1, \ldots, M
\end{array}
$$

where $\alpha_{\mathrm{i}}$ and $\beta_{\mathrm{i}}$ are weighting factors of positive and negative deviations, $\zeta_{\mathrm{i}}$ and $\tau_{i}$, of the $i$ th objective. The equality and inequality constraints of initial planning objectives, such as (10)-(18), are also included as the constraints of the composite objective. To ensure the obtained solution is minimally away from targets in either directions, $\alpha_{\mathrm{i}}$ and $\beta_{\mathrm{i}}$ are usually assigned the same values. The weighting factors of different objectives can be chosen according to the planner's preference in goal programming. A detailed discussion of planner preference in MOOP is given in Section V-A.

The proposed planning model is actually a mixed integer nonlinear programming (MINLP) problem with constraints, which is fairly difficult to be solved. In this paper, we use the branchand-bound (BB) method, which is a general searching method in global optimization and can efficiently solve the proposed planning model [20], [21]. The BB implementation starts with solving the relaxed nonlinear programming (NLP) of the original MINLP of (20). Subsequently, the feasible region of the NLP is "branched" recursively to form a tree of subproblems, according to the discrete variables, i.e., new circuit addition $\eta_{i j}$. By solving the subproblems within the tree, the optimal new circuit addition can be obtained. The advantage of the BB method is that the final solution is found with certainty to be optimal, though computational time may increase with the size of the problem [21], [22].

\section{SECURITY ASSESSMENT}

The expansion plan obtained from the MOOP approach does not realize any security constraints. Insecure configuration may exist in the resultant network. To ensure the system reliability, the expansion plan is assessed by a list of credible single line outages, i.e., the " $N-1$ " criteria, using an ac optimal load flow program. Should overload happen in any transmission line, the best individuals from the rest of the candidate pool are added into the network. This security assessment is repeated until no overloading happens in the system and the optimal expansion plan is finalized. The following presents the detailed procedure of the security assessment.
1) A list of line outages is selected using a contingency screening method based on the performance index (PI), which indicates the overloading in system [23]. To avoid "masking effect" [24], the selection is not stopped until no overload happens for two continuous outages. More details about PI-based contingency screening can be found in [23] and [24].

2) Based on the contingency list, a single line is removed in each outage case, and the power flow is calculated. The sum of line overloads in all outages is thus determined.

3) The best line to enhance reliability is selected from the rest of the candidate pool. The selection of the best candidate is based on a cost-benefit analysis of candidate line, i.e., the overload relieve per unit line construction cost defined as

$$
\begin{gathered}
O c_{l}=\frac{\Delta P_{l}}{C_{l}} \\
\text { where } \\
\Delta P_{l}=O V L_{B}-O V L_{A} .
\end{gathered}
$$

The line with the maximum $O c_{l}$ is considered the best candidate to be added in for system reliability purposes. Obviously, when $O V L_{B}$, total overload before new line addition, is zero, the expanded system is considered to be " $N-1$ " reliable, and the security assessment is terminated with final expansion plan obtained.

Note that step 3) above explains the case where adding one line is enough to ensure no overloads in all contingencies. If more than one line is to be added, (21) and (22) still can be used in selection. However, in order to consider combinatorial effect of line costs and their effects on system overloading, the terms $\Delta P_{l}$ and $C_{l}$ in those equations should be changed to the overload variation due to all added lines and their total cost accordingly, which will be further developed in our future research.

\section{EXAMPLES AND DISCUSSIONS}

\section{A. Case Study}

The IEEE five-machine, 14-bus system is used in the case study to test the proposed method. Presented in Fig. 3, the system consists of five generators at buses $1,2,3,6$, and 8 . The total generation capacity is $620 \mathrm{MW}$, and the initial load is $259 \mathrm{MW}$, distributed unevenly among the buses. Originally, the system has a total of 20 transmission circuits.

Two cases have been studied using MATLAB, which may demerit the work though with great simplicity in programming. The system loads are increased 2 and 2.2 times to $518 \mathrm{MW}$ and $570 \mathrm{MW}$, respectively, in the two cases, of which ac optimal power flow proved to diverge, due to overloads happening in transmission lines. The test program first selects the candidate circuits for network expansion as the initialization stage of the planning method, which is explained in Section III-A. These circuits have different characteristics. Given the proposed objectives for network planning, the important characteristics of those circuits include circuit distance, the expected profit rate of transmission, and maximum number of circuit to be expanded. Since those parameters are not with the original 14-bus system, we 


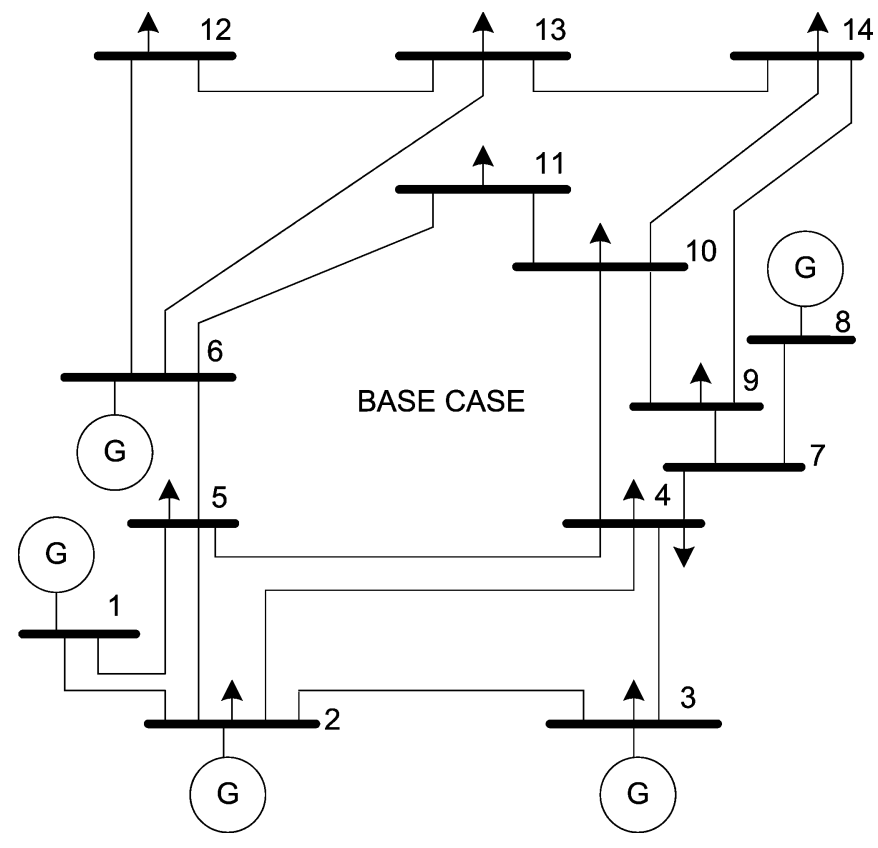

Fig. 3. IEEE five-machine 14-bus system.

have carefully designed the data for our case study as in the Appendix, which does not necessarily resemble the actual system.

The desired targets in (20) are selected by optimizing objective (7)-(9) independently. To ensure the solution of MOOP is minimally away from the target in either direction, positive and negative deviation factors $\alpha_{\mathrm{i}}$ and $\beta_{\mathrm{i}}$ in (20) are assigned the same values for each objective, and all weighting factors are assigned unit value, assuming all objectives are equally important to the planner.

From the MOOP procedure, the initial expansion plans are generated for both cases. The result shows that candidate routes 3 and 20 are selected for Case 1 and candidate routes 3, 12, 15, and 20 are selected for Case 2. Subsequently, the security assessment (SA) is carried out, and two extra lines are added in both cases to ensure the system reliability. Fig. 4 graphically presents the final expansion plans for the two cases studied, where dash lines represent the new lines to be added. Table I summarizes the simulation results of the two cases, where maximum addition of each transmission route is also given. Generally, Case 1 needs M $\$ 8.10$ for expanding $400 \mathrm{MW}$ of transmission capacity, while Case 2 needs M\$13.10 for expansion of $600 \mathrm{MW}$. Comparing the results before and after security assessment, both of the two cases need extra lines and corresponding investment for security enhancement, revealing the fundamental confliction between economy and security in planning expansion. It is also noted that Case 2 has selected more candidates for expansion than Case 1, which can be explained by the higher increase in system demand.

In addition, dispatches of an ideal SMP-based market have been simulated by running optimal power flows of the system studied in Case 1. Fig. 5 compares the resultant real power flows (absolute value) in transmission routes before and after expansion. The capacity limit of each route is also presented. It can be seen that power flow distribution has been largely changed due to network expansion, which benefits the system with improved
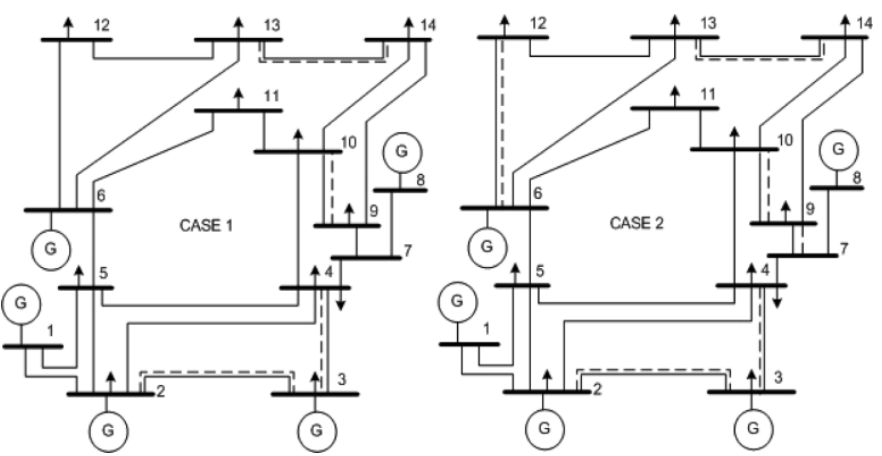

Fig. 4. Final expansion plans of Case 1 and 2 (dashed lines represent new lines expanded).

TABLE I

SimUlation Results In CASE StUdy

\begin{tabular}{lccccc}
\hline \hline \multirow{2}{*}{ Route } & Max & \multicolumn{2}{c}{ Case 1 } & \multicolumn{2}{c}{ Case 2 } \\
& expansion & Before SA & After SA & Before SA & After SA \\
\hline 3 & 1 & 1 & 1 & 1 & 1 \\
6 & 1 & 0 & 1 & 0 & 1 \\
11 & 2 & 0 & 0 & 0 & 0 \\
12 & 1 & 0 & 0 & 1 & 1 \\
15 & 1 & 0 & 0 & 1 & 1 \\
16 & 1 & 0 & 1 & 0 & 1 \\
18 & 1 & 0 & 0 & 0 & 0 \\
20 & 2 & 1 & 1 & 1 & 1 \\
Total investment(M\$) & 5.20 & 8.10 & 10.20 & 13.10 \\
Total capacity added (MW) & 200 & 400 & 400 & 600 \\
\hline \hline
\end{tabular}

security margin of transmission capacity. Especially, the flow in route 3 is about its limit of $100 \mathrm{MW}$ before expansion. With one line added, the flow has increased by over $1 / 3$ of the previous value given the same system demand, which is much lower than its new limit of $200 \mathrm{MW}$, indicating the route's importance in power delivery and the necessity of its expansion.

\section{B. Discussion}

1) Planning Objectives: The proposed planning method basically is a static planning method, considering single configuration of generation and system demand, forecasted into the time horizon of planning. However, given the many uncertainties involved in forecasting, especially in the long term, it would be practical to consider various demand patterns, as well as its timing, in transmission planning. Thus, the proposed method can be modified. Particularly, the planning objectives (8) and (9) can be extended to include more system loading scenarios and their estimated durations as shown in (23) and (24). However, it should be pointed out that the problem will become large and difficult to solve accordingly

$$
\begin{aligned}
& F_{2}^{\prime}=\sum_{k=1}^{N_{D}}\left[\frac{f_{2}\left(\boldsymbol{P}_{G}^{K}\right) T_{k}}{(1+\mu)^{h_{k}+0.5}}\right] \\
& F_{3}^{\prime}=\sum_{k=1}^{N_{D}}\left[\frac{f_{3}\left(\boldsymbol{f}^{k}, \boldsymbol{\eta}, \boldsymbol{r}, \boldsymbol{d}\right) T_{k}}{(1+\mu)^{h_{k}+0.5}}\right] .
\end{aligned}
$$

Similarly, transmission losses in dollar terms can also be included as one goal to be minimized. In addition, to further account for the stochastic nature of power system operation and planning, probabilistic indexes can be incorporated into the 


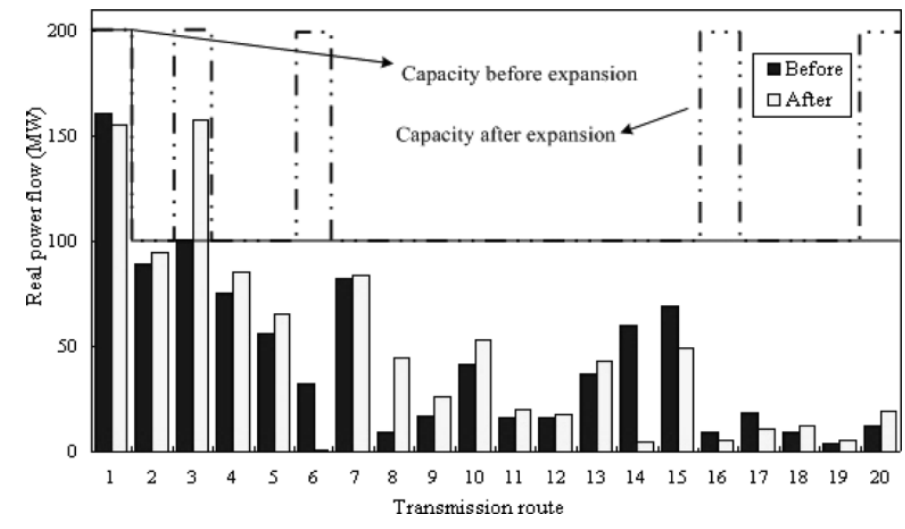

Fig. 5. Power flow distribution of Case 1 before and after expansion.

planning objectives, as well as the security assessment, of the proposed method in our future scope [25].

2) Planner's Preference in Planning: The MOOP in the proposed method is actually a preference-based technique [14]. In the case study, we assume that the planner would have equal preference in each objective. In practice, this will depend on the planner's role in system operation and planning. If a Transco or private investor is the planner, the priority would be both investment and profit prospect. If the ISO plans the expansion, operation cost and investment would be preferred. These can be done through adjusting weighting factors of different objectives in (20), as shown in Fig. 6, where the shaded area represents a portion of the objective space of a minimization problem with three objectives, and the Pareto front (surface) represents the collection of all Pareto-optimal solutions. Point $Z^{*}$ is the desired target, and $Z 1$ is Pareto-optimal solutions with equal preference in all three objectives and $Z 2$ has biased preference to $F 2$ and $F 3$ with relaxation to $F 1$.

In most European countries, the ISO actually owns and runs the transmission network as the so-called transmission system operator (TSO) [1]. In such cases, all three objectives in the proposed planning method would be equally concerned as in the presented case studies.

\section{CONCLUSION}

The deregulation process has introduced new challenges and uncertainties into the power system. This paper identifies that the complexity of the transmission planning in a deregulated environment requires new techniques. Traditional planning methods fail to account for the new appearing factors and therefore are no longer appropriate. To jointly cope with the increasing uncertainties and challenges in electricity market operation and planning, a hybrid multi-objective planning method for transmission expansion with security assessment is proposed. Multiple market operation and planning objectives are tackled simultaneously in the proposed approach by using the goal programming technique. The work presented in this paper is still at its initial stage of our continuous development of new planning techniques for deregulated power systems, in which we believe the hybrid MOOP approach is an appropriate

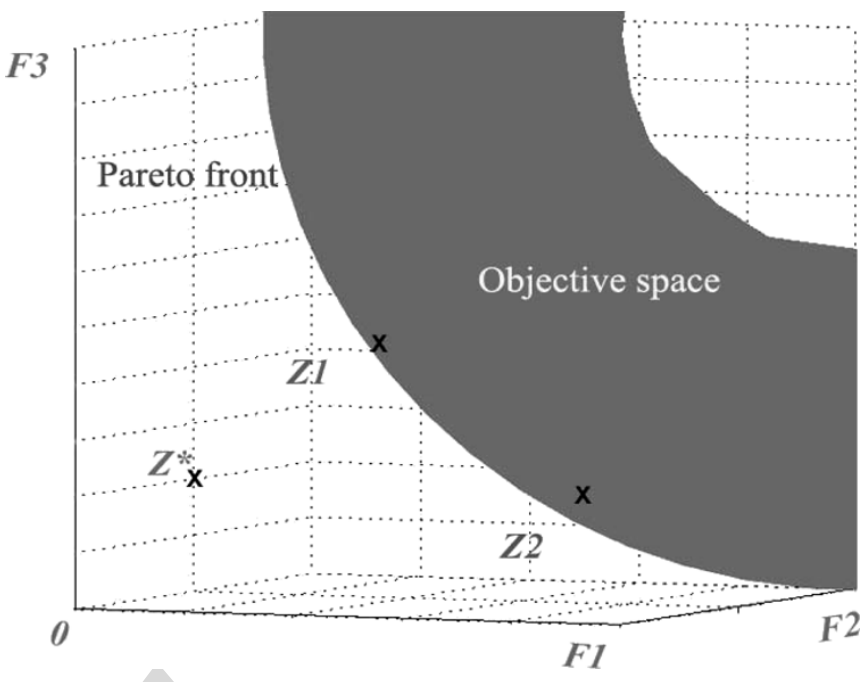

Fig. 6. Illustration of the influence of planner's preference in MOOP.

technique and has more potential in dealing with various uncertainties appeared with deregulation. Further development of the proposed method is underway to incorporate probabilistic indexes and additional realistic factors in market operation, such as the transmission congestion and losses, into the planning objective as well as reliability assessment. Furthermore, the method will be compared with other methods to further evaluate its effectiveness and economic impacts in the long term.

\section{APPENDIX}

Author: Please cite Tables II, III, and IV within the text.

TABLE II

TRANSMISSION LINE DATA (BASEMVA 100)

\begin{tabular}{ccccc}
\hline \hline No & Buses & $\begin{array}{c}\text { Reactance } \\
\text { p.u. }\end{array}$ & $\begin{array}{c}\text { Resistance } \\
\text { p.u. }\end{array}$ & $\begin{array}{c}\text { Capacity } \\
\text { (MVA) }\end{array}$ \\
\hline 1 & $1-2$ & 0.05917 & 0.01938 & 200 \\
2 & $1-5$ & 0.22304 & 0.05403 & 100 \\
3 & $2-3$ & 0.19797 & 0.04699 & 100 \\
4 & $2-4$ & 0.17632 & 0.05811 & 100 \\
5 & $2-5$ & 0.17388 & 0.05695 & 100 \\
6 & $3-4$ & 0.17103 & 0.06701 & 100 \\
7 & $4-5$ & 0.04211 & 0.01335 & 100 \\
8 & $4-7$ & 0.2045 & 0.00000 & 100 \\
9 & $4-9$ & 0.5389 & 0.00000 & 100 \\
10 & $5-6$ & 0.2349 & 0.00000 & 100 \\
11 & $6-11$ & 0.1989 & 0.09498 & 100 \\
12 & $6-12$ & 0.25581 & 0.12291 & 100 \\
13 & $6-13$ & 0.13027 & 0.06615 & 100 \\
14 & $7-8$ & 0.17615 & 0 & 100 \\
15 & $7-9$ & 0.11001 & 0 & 100 \\
16 & $9-10$ & 0.0845 & 0.03181 & 100 \\
17 & $9-14$ & 0.27038 & 0.12711 & 100 \\
18 & $10-11$ & 0.19207 & 0.08205 & 100 \\
19 & $12-13$ & 0.19988 & 0.22092 & 100 \\
20 & $13-14$ & 0.34802 & 0.17093 & 100 \\
\hline \hline
\end{tabular}


TABLE III

CANDIDATE TRANSMISSION ROUTES FOR EXPANSION

\begin{tabular}{ccccc}
\hline \hline No & $\begin{array}{c}\text { Max } \\
\text { expansions }\end{array}$ & $\begin{array}{c}\text { Distance } \\
\text { (Mile) }\end{array}$ & $\begin{array}{c}\text { Profit rate } \\
\text { (\$/MW-mile) }\end{array}$ & $\begin{array}{c}\text { Construction Cost } \\
\text { (M\$) }\end{array}$ \\
\hline 3 & 1 & 10.0 & 5.00 & 2.00 \\
6 & 1 & 8.5 & 4.25 & 1.60 \\
11 & 2 & 10.0 & 5.00 & 1.80 \\
12 & 1 & 13.0 & 6.50 & 1.90 \\
15 & 1 & 5.5 & 2.75 & 3.10 \\
16 & 1 & 4.0 & 2.00 & 1.30 \\
18 & 1 & 9.5 & 4.75 & 1.80 \\
20 & 2 & 17.5 & 8.75 & 3.20 \\
\hline \hline
\end{tabular}

TABLE IV

GENERATION BUSES DATA

\begin{tabular}{ccccc}
\hline \hline Bus & Generation Capacity, MW & $a_{i}$ & $b_{i}$ & $c_{i}$ \\
\hline 1 & 250 & 100 & 10 & 0.01 \\
2 & 200 & 100 & 10 & 0.01 \\
3 & 60 & 100 & 20 & 0.02 \\
6 & 50 & 100 & 15 & 0.02 \\
8 & 60 & 100 & 45 & 0.03 \\
\hline \hline
\end{tabular}

\section{ACKNOWLEDGMENT}

The authors would like to thank Dr. L. Shi for the valuable input from discussions.

\section{REFERENCES}

[1] K. Bhattacharya, M. H. J. Bollen, and J. E. Daalder, Operation of Reconstructed Power System. Norwell, MA: Kluwer, 2001.

[2] F. F. Wu and P. Varaiya, "Coordinated multilateral trades for electric power networks: Theory and implementation 1," Elect. Power Energy Syst., vol. 21, pp. 75-102, 1999.

[3] A. H. El-Abiad, "Power systems analysis and planning," in Proc. Arab School Science Technology, Kuwait, Jan. 21-29, 1981.

[4] X. F. Wang, Fundamentals of Power System Planning, China: China Electric Power, 1993.

\section{Author: City of publisher?}

[5] H. A. Gil and E. L. da Silva, "A reliable approach for solving the transmission network expansion planning problem using genetic algorithm," Elect. Power Syst. Res., vol. 58, pp. 45-51, 2001.

[6] M. V. F. Pereira and L. M. V. G. Pinto, "Application of sensitivity analysis of load supplying capacity to interactive transmission expansion planning," IEEE Trans. Power App. Syst., vol. PAS-104, pp. 381-389, Feb. 1985.

\section{Author: Issue number?}

[7] R. Romero and A. Monticelli, "A hierarchical decomposition approach for transmission network expansion planning," IEEE Trans. Power Syst., vol. 9, no. 1, pp. 373-380, Feb. 1994.

[8] R. Romero, R. A. Gallego, and A. Monticelli, "Transmission system expansion planning by simulated annealing," IEEE Trans. Power Syst., vol. 11, no. 1, pp. 364-369, Feb. 1996.

[9] E. L. Silva, H. A. Gil, and J. M. Areiza, "Transmission network expansion planning under an improved genetic algorithm," IEEE Trans. Power Syst., vol. 15, no. 3, pp. 1168-1175, Aug. 2000.

[10] R. A. Gallego, R. Romero, and A. J. Monticelli, "Tabu search algorithm for network synthesis," IEEE Trans. Power Syst., vol. 15, no. 2, pp. 490-495, May 2000.
[11] “Transmission Planning in an Open Access Environment," EPRI, Palo Alto, CA, Tech. Rep. 108 215, 1997.

[12] W. Wong, H. Chao, D. Julian, P. Lindberg, and S. Kolluri, "Transmission planning in a deregulated environment," in Proc. IEEE Transmission Distribution Conf., vol. 11-16, Apr. 1999, pp. 350-355.

[13] A. K. David and F. S. Wen, "Transmission planning and investment under competitive electricity market environment," in Proc. IEEE PES Summer Meeting, vol. 3, Jul. 15-19, 2001, pp. 1725-1730.

[14] K. Deb, Multi-Objective Optimization using Evolutionary Algorithm. New York: Wiley, 2001.

[15] Z. Xu, Z. Y. Dong, and W. Q. Liu, "Short-term electricity price forecasting using wavelet and SVM techniques," in Proc. 3rd Int. DCDIS Conf. Engineering Applications Computational Algorithms, Guelph, ON, Canada, May 15-18, 2003.

[16] Z. Xu and Z. Y. Dong, "A multi-resolution neural network model for electricity market price forecasting," in Proc. 4th Workshop Signal Processing Applications (WOSPA), Brisbane, Australia, Dec. 17-18, 2002.

[17] S. Stoft, Power System Economics. Piscataway, NJ: IEEE Press, 2002.

[18] D. Shirmohammadi, C. Rajagopalan, E. R. Alward, and C. L. Thomas, "Cost of transmission transactions: An introduction," IEEE Trans. Power Syst., vol. 6, no. 4, pp. 1546-1560, Nov. 1991.

[19] D. Shirmohammadi, X. V. Filho, B. Gorenstin, and M. V. P. Pereira, "Some fundamental technical concepts about cost-based transmission pricing," IEEE Trans. Power Syst., vol. 11, no. 2, pp. 1002-1008, May 1996.

[20] C. A. Floudas, Nonlinear and Mixed Integer Optimization: Fundamentals and Applications. London, U.K.: Oxford Univ. Press, 1995.

[21] S. Gören. Branch-and-Bound Procedures and Beam Search in Scheduling. LORS: Library of Online Resources on Scheduling [Online]Available: http://www.ie.bilkent.edu.tr/ lors/lors/method.html.

[22] S. Leyffer, "Integrating SQP and branch-and-bound for mixed integer nonlinear programming," Comput. Optim. Appl., vol. 18, pp. 295-309, 2001.

[23] T. A. Mikolynnas and B. F. Wollenberg, "An advanced contingency selection algorithm,” IEEE Trans. Power App. Syst., vol. PAS-100, no. 2, 1981.

\section{Author: Month?}

[24] K. F. Schafer and J. F. Verstege, "Adaptive procedure for masking effect compensation in contingency selection algorithms," IEEE Trans. Power Syst., vol. 5, no. 2, pp. 539-546, May 1990.

[25] "Moving Toward Probabilistic Reliability Assessment Methods: A Framework for Addressing Uncertainty in Power System Planning and Operation,” EPRI, Palo Alto, CA, Tech. Rep. 1002 639, Dec. 2003.
Zhao Xu (S'00) received the B.Eng. degree in electrical engineering from Zhejiang University, China, Author: In what city? in 1996, and the M.Eng. degree in electrical engineering from the National University of Singapore in $2002 . \mathrm{He}$ is currently working toward the Ph.D. degree at the University of Queensland, St. Lucia, Australia.

$\mathrm{He}$ has been with Huaneng Power International and ABB. His research interests include electricity market planning and management, power system stability, and AI applications.

Zhao Yang Dong (M'99) received the Ph.D. degree in electrical and information engineering from the University of Sydney, Sydney, Australia, in 1999.

$\mathrm{He}$ is now a Senior Lecturer at the School of Information Technology and Electrical Engineering, the University of Queensland, St. Lucia, Australia. His research interest includes power system security assessment and enhancement, electricity market, artificial intelligence and its application in electric power engineering, power system planning and management, and power system stability and control. 
Kit Po Wong (M'87-SM'90-F'02) received the M.Sc., Ph.D., and D.Eng. degrees from the University of Manchester, Institute of Science and Technology, Manchester, U.K., in 1972, 1974, and 2001, respectively.

He was with the University of Western Australia beginning in 1974. Currently, he is a Chair Professor and Head of the Department of Electrical Engineering, the Hong Kong Polytechnic University, Kowloon, Hong Kong. His research interests include artificial intelligence and evolutionary computation applications to power system planning and operations.

Prof. Wong received three Sir John Madsen Medals (1981, 1982, and 1988) from the Institution of Engineers Australia, the 1999 Outstanding Engineer Award from IEEE Power Chapter Western Australia, and the 2000 IEEE Third Millennium Award. He was a Co-Technical Chairman of the IEEE ICMLC 2004 Conference and General Chairman of IEEE PowerCon2000. He is an Honorary Editor of IEE PROCEEDINGS IN GENERATION, TRANSMISSION AND DistRIBUTION and Editor (Electrical) of the Transactions of Hong Kong Institution of Engineers. He is a Fellow of IEE, HKIE, and IEAust. 